\title{
Non-meagre subgroups of reals disjoint with meagre sets
}

\author{
Ziemowit Kostana \\ Faculty of Mathematics, Informatics and Mechanics \\ Warsaw University \\ 02-097 Warszawa, Poland \\ E-mail: z.kostana@mimuw.edu.pl
}

10.2017

\begin{abstract}
Let $(X,+)$ denote $(\mathbb{R},+)$ or $\left(2^{\omega},+_{2}\right)$. We prove that for any meagre set $F \subseteq X$ there exists a subgroup $G \leq X$ without the Baire property, disjoint with some translation of $F$. We point out several consequences of this fact and indicate why analogous result for the measure cannot be established in ZFC. We extend proof techniques from [1].
\end{abstract}

\section{Historical Background}

For sets $A, B \subseteq \mathbb{R}$ we define the algebraic sum $A+B=\{a+b \mid a \in A, b \in B\}$. Study of algebraic sums of this kind has been around for almost a century. The first result in this topic seems to be due to Sierpiński, who proved in 1920 that there exists two sets of measure zero whose sum is non-measurable [4]. Rubel [12] showed that these two sets can be chosen to be equal. This result was later generalized in many directions. For example, related results for other $\sigma$-ideals were obtained by Kharazishvili [10] and by Cichoń and Jasiński [5]. In another direction, Ciesielski, Fejzić and Freiling [6] proved among others, that for every set $C \subseteq \mathbb{R}$, there exists a set $A \subset C$ such that $\lambda_{*}(A+A)=0$ and $\lambda^{*}(A+A)=\lambda^{*}(C+C)$, where $\lambda_{*}$ and $\lambda^{*}$ denote the inner and the outer Lebesgue measure respectively (but for simpler proof

2010 Mathematics Subject Classification: Primary 28A05, 54E52.

Key words and phrases: non-measurable subgroup, Baire property, algebraic sum. 
see the work by Marcin Kysiak [13]). It is also worth to mention the famous Erdös-Kunen-Mauldin theorem [9].

It is easy to see that the sum of compact (open) sets is compact (open), the sum of $F_{\sigma}$ sets is $F_{\sigma}$, but for higher Borel classes this is not the case [5]. Even the sum of a compact set with $G_{\delta}$ doesn't have to be a Borel set, and this was shown by Sodnomow in 1954 [11] and independently by Erdös and Stone in 1970 [8].

The study of algebraic sums of subsets of real line is closely related to the study of additive subgroups of $(\mathbb{R},+)$. Erdös proved, that under $\mathrm{CH}$ there exists a non-meagre, null additive subgroup of reals, as well as a nonmeasurable, meagre additive subgroup of reals [7]. The same can be proved under MA, but somehow surprisingly, while non-meagre subgroups of measure zero always exists, some additional set-theoretic assumption turns out to be necessary to prove the existence of a subgroup which is non-measurable and meagre. This was proved recently by Rosłanowski and Shelah [1].

Results of this paper were obtained during the work on the author's Master's Thesis. Author would like to thank his advisor, dr. Marcin Kysiak, as well as prof. Witold Marciszewski and prof. Piotr Zakrzewski for many helpful remarks and suggestions.

\section{Preliminaries}

In private communication Sergei Akbarov posed the following problem, sometimes referred to as the Akbarov Problem.

Problem 1. Let $A \subseteq \mathbb{R}$ be a nonempty null set. Does there exist a set $B \subseteq \mathbb{R}$ with the property that $A+B$ is Lebesgue non-measurable?

One of the natural ways to approaching such problem is to try to find a non-measurable dense subgroup $G \leq \mathbb{R}$ disjoint with some translation of $A$. Indeed, assume we have a non-measurable dense subgroup $G \leq \mathbb{R}$, and $(A+v) \cap G=\emptyset$ holds. Then also

$$
\begin{gathered}
(A+v) \cap(G-G)=\emptyset, \\
(A+v+G) \cap G=\emptyset .
\end{gathered}
$$

It is well-known (see for example [3], Thm. 7.36) that every dense subgroup of $\mathbb{R}$ is either null or has full outer measure. Both $G$ and $A+v+G$ have full outer measure, hence both have inner measure zero, and so are nonmeasurable.

This approach, however sufficient to solve the problem with certain additional assumptions like Martin's Axiom, won't work in ZFC alone. In 2016 Andrzej Rosłanowski and Saharon Shelah proved the following theorem [1].

Theorem 1. It is relatively consistent with $Z F C$ that any meagre subgroup of reals is null. 
Indeed, consider a dense $G_{\delta}$ null subset of reals. Any subgroup disjoint with its translation must be meagre. So, consistently, also null.

In the case of Baire category however, situation is different. The following is the main result of this paper.

Main Theorem. Let $X=(\mathbb{R},+)$ or $X=\left(2^{\omega},+_{2}\right)$. For any meagre set $F \subseteq X$, there exists $x \in X$, and a dense subgroup $H \leq X$ without the Baire property such that $(F+x) \cap H=\emptyset$.

From this follows the affirmative answer to the category version of Problem 1.

Corollary 1. For any meagre set $A \subseteq X$, there exist a set $B \subseteq X$ such that $A+B$ doesn't have the Baire property.

Proof. Just take as $B$ a dense subgroup without the Baire property, which is disjoint with a translation of $A$.

Another consequence is

Corollary 2. There exists a null subgroup of $X$ which is not meagre.

This was firstly proved by Talagrand [15], and more recently by Rosłanowski and Shelah [1].

Proof. Just take $F$ in Theorem 2 of full measure.

In the whole paper $X$ denote the group of reals $(\mathbb{R},+)$ or the Cantor space $\left(2^{\omega},+_{2}\right)$ with coordinate-wise addition modulo 2 . Instead of $A+\{x\}$, we write $A+x . \mathcal{M}$ and $\mathcal{N}$ denote $\sigma$-ideals of meagre and null sets respectively. A partition of $\omega$ is always a partition on finite intervals.

The following quantifiers are commonly used in the infinite combinatorics:

- $\forall_{n<\omega}^{\infty} \psi(n)$, denoting " $\psi$ holds for sufficiently large $n$ ";

- $\exists_{n<\omega}^{\infty} \psi(n)$, denoting " $\psi$ holds for infinitely many $n$ ".

Here, one more similar notation will prove useful. Let $\mathfrak{U}$ be a fixed nonprincipial ultrafilter on $\omega$. Expression

$$
\mathfrak{U}_{n<\omega} \psi(n)
$$

will mean

$$
\{n \mid \psi(n)\} \in \mathbb{U} .
$$

$\mathfrak{U}$ can be seen as something between $\forall^{\infty}$, and $\exists^{\infty}$. If $\forall_{n<\omega}^{\infty} \psi(n)$ and $\forall_{n<\omega}^{\infty} \phi(n)$, then clearly $\forall_{n<\omega}^{\infty} \psi(n) \wedge \phi(n)$, and this is not the case for $\exists^{\infty}$. On the other hand, for any $\psi$ either $\exists_{n<\omega}^{\infty} \psi(n)$ or $\exists_{n<\omega}^{\infty} \neg \psi(n)$, and this is not the case for $\forall^{\infty}$. It is straightforward from the definition of a non-principial ultrafilter that for $\mathfrak{U}$ both mentioned conditions holds.

The following combinatorial characterization of meagre sets in $2^{\omega}$, due to Bartoszyński (for the proof see [2], Thm. 2.2.4), will be crucial in our considerations. 
Theorem 2. Every meagre subset of $2^{\omega}$ is contained in a meagre set of the form

$$
F=\left\{x \in 2^{\omega} \mid \forall_{n<\omega}^{\infty} x \uparrow I_{n} \neq v \uparrow I_{n}\right\},
$$

where $\left\{I_{n}\right\}_{n<\omega}$ is a partition of $\omega$, and $v \in 2^{\omega}$.

Remark. It is not hard to see, that once we have the partition $\left\{I_{n}\right\}_{n<\omega}$, we can replace it with a "thicker" partition, i.e. one in which the end of every interval lies in some fixed infinite subset of $\omega$.

Historical Note. H. Friedman and S. Shelah independently proved, that if a model $V$ results from adding $\omega_{2}$ Cohen reals to a model of $C H$, then in $V$ the following holds: if $E$ is an $F_{\sigma}$ subset of $X \times X$, which contains a rectangle of positive outer measure, then it contains a rectangle of positive measure. From this, M. Burke [16] concludes Theorem 1 as a corollary. For another reference, see [14].

\section{Main theorem}

We turn to the proof of our main theorem. Firstly, we prove it for $X=$ $2^{\omega}$, and then for $X=\mathbb{R}$, which will turn out to be more complicated. The following lemma was implicitly used in [1] to obtain null, non-meagre subgroup of the Cantor Space, but in fact, there's more we can get from it.

Lemma 1. Let $\left\{I_{n}\right\}_{n<\omega}$ be a partition. Then $G=\left\{x \in 2^{\omega} \mid \mathfrak{u}_{n<\omega} x \uparrow I_{n} \equiv 0\right\}$ is a non-meagre dense subgroup of $2^{\omega}$.

Proof. The fact that $G$ is a group is straightforward from properties of the ultrafilter. It is dense, since every sequence eventually equal 0 is in $G$. The non-trivial part is to show that it doesn't have the Baire property. It is well-known (see for example [3], Thm. 7.38) that dense, proper subgroups of $2^{\omega}$ which have the Baire property are meagre, so it's enough to show that group $G$ is not meagre. Consider any $M \in \mathcal{M}\left(2^{\omega}\right)$. By virtue of Theorem 2 we can assume, possibly enlarging $M$, that

$$
M=\left\{x \in 2^{\omega} \mid \forall_{k<\omega}^{\infty} x \uparrow J_{k} \neq v \uparrow J_{k}\right\},
$$

for some $v \in 2^{\omega}$ and a partition $\left\{J_{n}\right\}_{n<\omega}$. Moreover, applying Remark after Theorem 2, we can choose intervals $J_{k}$ in such a way that each of them is a finite sum of consecutive intervals of the form $I_{r}$, like below.

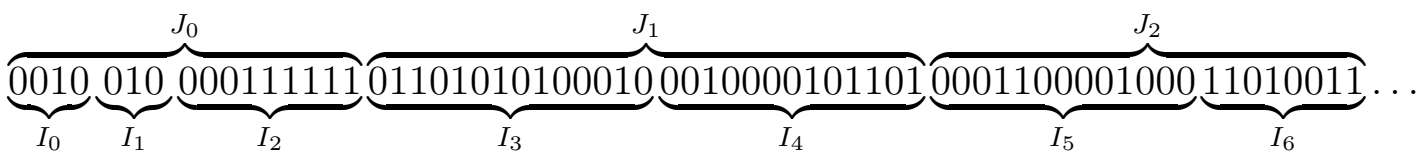

Let now

$$
A_{0}=\left\{n<\omega \mid \exists_{r<\omega} I_{n} \subseteq J_{2 r}\right\}
$$


and

$$
A_{1}=\left\{n<\omega \mid \exists_{r<\omega} I_{n} \subseteq J_{2 r+1}\right\} .
$$

One of these sets belongs to the ultrafilter $\mathfrak{U}$. Suppose it's $A_{0}$. Then we put

$$
x \uparrow I_{k}= \begin{cases}0, & \text { if } k \in A_{0} \\ v \uparrow I_{k}, & \text { if } k \in A_{1} .\end{cases}
$$

Similarily, if $A_{1} \in \mathfrak{U}$, we put

$$
x \uparrow I_{k}= \begin{cases}0, & \text { if } k \in A_{1} \\ v \uparrow I_{k}, & \text { if } k \in A_{0} .\end{cases}
$$

In any case, $x$ is constructed in such a way that $\mathfrak{U}_{n<\omega} x \uparrow I_{n} \equiv 0$, but also $\exists_{n<\omega}^{\infty} x \uparrow J_{n}=v \uparrow J_{n}$. In fact, $\left\{n<\omega \mid x \uparrow J_{n}=v \uparrow J_{n}\right\}$ is either the set of even or the set of odd non-negative integers. This means that $x \in G \backslash M$, and since $M$ was arbitrary, this shows that $G \notin \mathcal{M}$.

Theorem 3. For any set $F \in \mathcal{M}$, there exist a $x \in X$ and a dense subgroup $H \leq X$ without the Baire property such that $(F+x) \cap H=\emptyset$.

\subsection{Version for $2^{\omega}$}

Take any $F \in \mathcal{M}\left(2^{\omega}\right)$. Using Theorem 2 , we can assume that

$$
F=\left\{x \in 2^{\omega} \mid \forall_{k<\omega}^{\infty} x \uparrow I_{k} \neq x_{F} \uparrow I_{k}\right\}
$$

where $x_{F} \in 2^{\omega}$, and $\left\{I_{n}\right\}_{n<\omega}$ is a partition of $\omega$. Then, we only have to notice that $\left\{x \in 2^{\omega} \mid \mathfrak{U}_{n<\omega} x \uparrow I_{n} \equiv 0\right\} \cap\left(F+{ }_{2} x_{F}\right)=\emptyset$ and use Lemma 1 . To this end, see that

$$
\left\{x \in 2^{\omega} \mid \mathfrak{H}_{n<\omega} x \uparrow I_{n} \equiv 0\right\} \subseteq\left\{x \in 2^{\omega} \mid \exists_{n<\omega}^{\infty} x \uparrow I_{n} \equiv 0\right\}
$$

and

$$
F+{ }_{2} x_{F} \subseteq\left\{x \in 2^{\omega} \mid \forall_{n<\omega}^{\infty} x \uparrow I_{n} \neq 0\right\}
$$

\subsection{Version for $\mathbb{R}$}

For any (finite or infinite) binary sequence $x$, we denote by $x^{o p}$ the sequence obtained from $x$ by changing every 0 to 1 , and vice versa. Let $D^{\infty}=\left\{x \in 2^{\omega} \mid \exists_{n<\omega}^{\infty} x(n)=0\right\}$. It is known that there exists the continuous bijection preserving measure and category, $\phi: D^{\infty} \rightarrow[0,1)$, given by the formula

$$
\phi(x)=\sum_{i=0}^{\infty} \frac{x(i)}{2^{i+1}}
$$


Consider any $F \in \mathcal{M}(\mathbb{R})$. Replacing if needed $F$ by $F+\mathbb{Z}$, we can assume that $F+\mathbb{Z}=F$. Let $\widetilde{F}=\phi^{-1}[F \cap[0,1)]$. There exists a $w \in 2^{\omega}$ and a partition of $\omega,\left\{I_{n}\right\}_{n<\omega}$, with the property that

$$
\widetilde{F} \subseteq\left\{x \in 2^{\omega} \mid \forall_{n<\omega}^{\infty} x \uparrow I_{n} \neq w \uparrow I_{n}\right\} .
$$

In this case, we'll need a combinatorial characterization with some stronger properties. We choose another partition of $\omega,\left\{J_{n}\right\}_{n<\omega}$, satisfying the following conditions:

- each interval $J_{n}$ is a sum of at least three consecutive intervals $I_{k}$;

- if $J_{n}=I_{r_{0}} \cup \ldots \cup I_{r_{k}}$, and indices are increasing, then $\left|I_{r_{2}} \cup \ldots \cup I_{r_{k}}\right|>\left|I_{r_{0}} \cup I_{r_{1}}\right|$

- sequence $\left\{\left|J_{n}\right|\right\}_{n<\omega}$ is increasing.

We now construct the sequence $v \in 2^{\omega}$. For every $n<\omega$, we write

$$
J_{n}=I_{r_{0}} \cup \ldots \cup I_{r_{k}}
$$

as a sum of consecutive intervals of the form $I_{k}$, with increasing indices, and put:

$$
\begin{gathered}
v \uparrow I_{r_{0}}=w \uparrow I_{r_{0}}, \\
v \uparrow I_{r_{1}}=w^{o p} \uparrow I_{r_{1}}, \\
v \uparrow I_{r_{2}} \cup \ldots \cup I_{r_{k}}=010101 \ldots 01(0) .
\end{gathered}
$$

Given (3.1), it is evident that

$$
\widetilde{F} \subseteq\left\{x \in 2^{\omega} \mid \forall_{n<\omega}^{\infty} x \uparrow J_{n} \neq v \uparrow J_{n}\right\} .
$$

Let $J_{n}=\left[a_{n}, b_{n}\right]$ for every $n$.

Lemma 2. Assume we have sequences $x, y, z \in D^{\infty}$ such that $x \uparrow[a, b] \equiv 0$, $y(b)=0$, and $\phi(z)+\rho=\phi(x)+\phi(y)$, where $\rho \in\{0,1\}$. Then $y \uparrow[a, b-1]=$ $z \uparrow[a, b-1]$.

Proof.

$$
\begin{array}{r}
\phi(x)+\phi(y)=\sum_{i=0}^{a-1} \frac{x(i)}{2^{i+1}}+\sum_{i=a}^{b} \frac{x(i)}{2^{i+1}}+\sum_{i=b+1}^{\infty} \frac{x(i)}{2^{i+1}}+ \\
\sum_{i=0}^{a-1} \frac{y(i)}{2^{i+1}}+\sum_{i=a}^{b} \frac{y(i)}{2^{i+1}}+\sum_{i=b+1}^{\infty} \frac{y(i)}{2^{i+1}}= \\
\sum_{i=0}^{a-1} \frac{x(i)+y(i)}{2^{i+1}}+\sum_{i=a}^{b-1} \frac{y(i)}{2^{i+1}}+\sum_{i=b+1}^{\infty} \frac{x(i)+y(i)}{2^{i+1}}= \\
\sum_{i=0}^{\infty} \frac{z(i)}{2^{i+1}}+\rho .
\end{array}
$$


$\sum_{i=b+1}^{\infty} \frac{x(i)+y(i)}{2^{i+1}}<\sum_{i=b+1}^{\infty} \frac{1}{2^{i}}=2^{-b}$, so this part of the sum does not affect positions of $z$ with indices less than $b$.

Let us now define

$$
\widetilde{G}=\left\{x \in 2^{\omega} \mid \exists_{m<\omega} \mathfrak{u}_{n<\omega} x \uparrow\left[a_{n}, b_{n}-m\right] \equiv 0\right\} .
$$

In fact, we didn't rule out the possibility that $m>b_{n}-a_{n}$, but recall that $\left|a_{n}-b_{n}\right| \rightarrow \infty$, so this situation can only occur on finitely many positions. This is clearly a subgroup of $2^{\omega}$, and $\widetilde{G} \supseteq\left\{x \in 2^{\omega} \mid \mathfrak{H}_{n<\omega} x \uparrow J_{n} \equiv 0\right\}$, which, by Lemma 1 , is not meagre, so we obtain that $\widetilde{G}$ is not meagre.

Group $\widetilde{G}$ defined this way can be "transferred" to $\mathbb{R}$, and in fact authors of [1] apply this kind of idea.

Lemma 3. $\phi[\widetilde{G}]+\mathbb{Z}$ is a subsemigroup of $\mathbb{R}$.

Proof. Assume we have an equality

$$
\phi(g)+k_{1}+\phi(h)+k_{2}=\phi(f)+l
$$

where $g, h \in \widetilde{G}, k_{1}, k_{2}, l \in \mathbb{Z}$. We shall show that $f \in \widetilde{G}$.

There exist integers $m_{1}, m_{2}$ such that

$$
\mathfrak{u}_{n} g \uparrow\left[a_{n}, b_{n}-m_{1}\right] \equiv 0, \mathfrak{u}_{n} h \uparrow\left[a_{n}, b_{n}-m_{2}\right] \equiv 0,
$$

so that if we set $\bar{m}=\max \left\{m_{1}, m_{2}\right\}$, we obtain

$$
\mathfrak{u}_{n} g \uparrow\left[a_{n}, b_{n}-\bar{m}\right] \equiv h \uparrow\left[a_{n}, b_{n}-\bar{m}\right] \equiv 0
$$

Then, from Lemma 2 we conclude that $\mathfrak{u}_{n} f \uparrow\left[a_{n}, b_{n}-(\bar{m}+1)\right] \equiv 0$, which shows that $f \in \widetilde{G}$.

Lemma 4. $\widetilde{G}+{ }_{2} v, \widetilde{G}+{ }_{2} v^{o p} \subseteq D^{\infty}$, moreover $\phi\left[\widetilde{G}+{ }_{2} v\right]+\mathbb{Z} \supseteq \phi[\widetilde{G}]+\phi\left[\widetilde{G}+{ }_{2} v\right]+\mathbb{Z}$, and $\phi\left[\widetilde{G}+{ }_{2} v^{o p}\right]+\mathbb{Z} \supseteq \phi[\widetilde{G}]+\phi\left[\widetilde{G}+{ }_{2} v^{o p}\right]+\mathbb{Z}$.

Proof. The first part easily follows from the definitions. For the proof of the second part, assume we have a $z \in D^{\infty}$ with the property that $\phi(z)+\rho=$ $\phi\left(g_{1}\right)+\phi\left(g_{2}+{ }_{2} v\right)$, for some $g_{1}, g_{2} \in \widetilde{G}$ and $\rho \in\{0,1\}$. We'll show that $z \in \widetilde{G}+{ }_{2} v$.

Let $m_{1}, m_{2}<\omega$ satisfy

$$
\mathfrak{U}_{n<\omega} g_{i} \uparrow\left[a_{n}, b_{n}-m_{i}\right] \equiv 0,
$$

for $i=0,1$, and $\bar{m}=\max \left\{m_{1}, m_{2}\right\}$. Then

$$
\mathfrak{u}_{n<\omega}\left(g_{2}+{ }_{2} v\right) \uparrow\left[a_{n}, b_{n}-\bar{m}\right]=v \uparrow\left[a_{n}, b_{n}-\bar{m}\right]
$$


and

$$
\mathfrak{u}_{n<\omega} g_{1} \uparrow\left[a_{n}, b_{n}-\bar{m}\right] \equiv 0 .
$$

Because $\left|a_{n}-b_{n}\right| \rightarrow \infty$, for sufficiently large $n, \frac{a_{n}+b_{n}}{2} \leq b_{n}-\bar{m}$, which means that $b_{n}-\bar{m}$ lies in the right half of the interval $J_{n}$. Recall that $v$ was defined in such a way that in the right half of every interval $J_{n}$, it is of the form $0101010 \ldots 1(0)$. Thus for sufficiently large $n$, either $v\left(b_{n}-\bar{m}\right)=0$ or $v\left(b_{n}-(\bar{m}+1)\right)=0$. From Lemma 2 (putting $z=z, x=g_{1}, y=v$ ), we infer that

$$
\mathfrak{u}_{n<\omega} z\left\lceil\left[a_{n}, b_{n}-(\bar{m}+2)\right]=v \uparrow\left[a_{n}, b_{n}-(\bar{m}+2)\right] .\right.
$$

In conclusion $z+{ }_{2} v \in \widetilde{G}$, which is exactly $z \in \widetilde{G}+{ }_{2} v$. For $v^{o p}$ proof is the same, except that we replace every instance of $v$ with $v^{o p}$.

Notice now, that

$$
(\widetilde{G}+v) \cap \widetilde{F}=\emptyset .
$$

This is true, because of (3.1) and the inclusion

$$
(\widetilde{G}+v) \subseteq\left\{x \in 2^{\omega} \mid \exists_{n<\omega}^{\infty} x \uparrow I_{n}=w \uparrow I_{n}\right\} .
$$

Set $H=\phi[\widetilde{G}]-\phi[\widetilde{G}]+\mathbb{Z}$. From Lemma 3 directly follows that it is a subgroup of $\mathbb{R}$. What's left, is to verify that

$$
(\phi[\widetilde{G}]-\phi[\widetilde{G}]+\mathbb{Z}) \cap(F-\phi(v))=\emptyset .
$$

Suppose to the contrary, that for some $g, h \in \widetilde{G}, k \in \mathbb{Z}$ and $f \in F$, an equality

$$
\phi(g)-\phi(h)+\phi(v)+k=f
$$

holds. From Lemma 4 we know, that $\phi(g)+\phi(v)+k=\phi\left(g^{\prime}+{ }_{2} v\right)+k^{\prime}$; $g^{\prime} \in \widetilde{G}, k^{\prime} \in \mathbb{Z}$, so we obtain

$$
\phi\left(g^{\prime}+{ }_{2} v\right)-\phi(h)+k^{\prime}=f .
$$

One can verify that for any sequence $x \in D^{\infty}$, for which also $x^{o p} \in D^{\infty}$, identity $\phi(x)+\phi\left(x^{o p}\right)=1$ holds. This allows us to write

$$
\phi\left(g^{\prime}+{ }_{2} v\right)=1-\phi\left(\left(g^{\prime}+{ }_{2} v\right)^{o p}\right)=1-\phi\left(g^{\prime}+{ }_{2} v^{o p}\right),
$$

because $^{o p}$ operation is just the addition of a constant sequence, and so plugging (3.5) into (3.4) yields

$$
\begin{array}{r}
f=1-\phi\left(g^{\prime}+{ }_{2} v^{o p}\right)-\phi(h)+k^{\prime}= \\
1-\left[\phi\left(g^{\prime}+{ }_{2} v^{o p}\right)+\phi(h)\right]+k^{\prime}= \\
k^{\prime \prime}+1-\phi\left(\bar{g}+{ }_{2} v^{o p}\right)= \\
k^{\prime \prime}+\phi\left(\bar{g}+{ }_{2} v\right) .
\end{array}
$$


We used Lemma 4 in the third equality, and $\phi\left(\bar{g}+{ }_{2} v\right)+\phi\left(\bar{g}+{ }_{2} v^{o p}\right)=1$ in the last one. Since $F+\mathbb{Z}=F$, we conclude that

$$
\begin{gathered}
\phi\left(\bar{g}+{ }_{2} v\right) \in F \cap[0,1)=\phi[\widetilde{F}], \\
\bar{g}+{ }_{2} v \in \widetilde{F},
\end{gathered}
$$

which contradicts (3.3). Finally, let us notice that during the whole calculation arguments of $\phi$ were always within its domain, $D^{\infty}$.

\section{Open problems}

The proof of Theorem 2 uses the characterization of sets from the $\mathcal{M}\left(2^{\omega}\right)$ ideal, which turns out to match very well with the properties of ultrafilters. There's another class of sets with elegant combinatorial characterization, namely $\mathcal{E}$ - the $\sigma$-ideal generated by closed sets of measure zero. The following theorem is due to Bartoszyński and Shelah and is proved in [2].

Theorem 4. $E \in \mathcal{E}\left(2^{\omega}\right)$ if and only if

$$
E \subseteq\left\{x \in 2^{\omega} \mid \forall_{n<\omega}^{\infty} x \uparrow I_{n} \in K_{n}\right\}
$$

where $\left\{I_{n}\right\}_{n<\omega}$ is a partition of $\omega, K_{n} \subseteq 2^{I_{n}}$ and $\forall_{n<\omega} \frac{\left|K_{n}\right|}{2^{\left|I_{n}\right|}} \leq 2^{-n}$.

The following seems to be a reasonable question.

Problem 2. Let $E \in \mathcal{E}\left(2^{\omega}\right)$. Does there necessarily exists a dense nonmeasurable subgroup $G \leq 2^{\omega}$, disjoint with some translation of $E$ ?

Another related question was asked by Taras Banakh on the Mathoverflow.

Problem 3. Does there exist (in $Z F C$ ) a subgroup $G \leq X$, such that $G \in \mathcal{N} \cap \mathcal{M}$, but $G \notin \mathcal{E}$ ?

\section{References}

[1] Andrzej Rosłanowski, Saharon Shelah, Small-large subgroups of the reals, arXiv preprint arXiv:1605.02261, 2016

[2] Tomek Bartoszyński, Haim Judah, Set Theory: On the structure of the real line, A.K. Peters, Wellesley, 1995

[3] Lev Bukovský, The Structure of the Real Line, Springer Basel AG, 2011 
[4] W. Sierpiński, Sur la question de la measurabilité de la base de $M$. Hamel, Fund. Math., 1 (1920), p.105-111

[5] J. Cichon, A. Jasiński, A note on algebraic sums of subsets of the real line, Real Analysis Exchange, vol.28 (2002/2003), p.493-499

[6] K. Ciesielski, H. Fejzić, Ch. Freiling, Measure zero sets with nonmeasurable sum, Real Analysis Exchange, vol.27 (2001-2002), p.783793

[7] P. Erdös, Some remarks on subgroups of real numbers, Coll. Math., vol. XLII (1979), p.119-120

[8] P. Erdös and A. H. Stone, On the sum of two Borel sets, Proceedings of the American Mathematical Society vol.25 (1970), p.304-306

[9] P. Erdös, K. Kunen, and R. Mauldin, Some additive properties of sets of real numbers, Fund. Math., 113 (1981), no. 3, p.187-199

[10] A. Kharazishvili, Some remarks on additive properties of invariant $\sigma$ ideals on the real line, Real Analysis Exchange, vol.21 (1995-1996), p.715-724

[11] B. S. Sodnomow, Primier dwoch mnozestw tipa $G_{\delta}$, aritmeticzeskaja suma kotorych nieizmierima B, Doklady Akademii Nauk CCCR, 1954, vol. XCIX, No 4

[12] L. A. Rubel, A pathological Lebesgue measurable function, J. London Math. Soc., 38 (1963), 1-4

[13] M. Kysiak Nonmeasurable algebraic sums of sets of reals, Colloquium Mathematicum, vol. 102. (2005)

[14] J. Pawlikowski On a Rectangle Inclusion Problem, PAMS 123 (1995) 3189-3195

[15] M. Talagrand Compacts de fonctions mesurables et filtres nonmesurables, vol.67(1) (1980)

[16] M. Burke, A theorem of Friedman on rectangle inclusion and its consequences, Note of March 7, 1991,

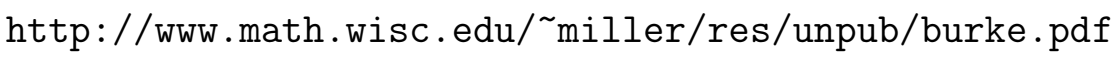

